Received: 05.04 .2018

Revised: 20.04 .2018

Accepted: 25.05 .2018

DOI: $10.17804 / 2410-9908.2018 .3 .006-013$

\title{
THE DIGITAL IMAGE CORRELATION METHOD APPLIED TO STUDYING THE PLASTIC FLOW OF THE 08G2BM STEEL UNDER TENSION
}

\author{
S. V. Smirnov ${ }^{1, \text { a) }}$, V. A. Khotinov ${ }^{2, \text { b) }}{ }_{*}$, D. I. Vichuzhanin ${ }^{1, c)}$, \\ O. N. Polukhina, ${ }^{2, \text { d) }}$, V. M. Farber ${ }^{2, \text { e) }}$ \\ ${ }^{I}$ Institute of Engineering Science, Ural Branch of the Russian Academy of Sciences, \\ 34 Komsomolskaya St., Ekaterinburg, Russian Federation \\ ${ }^{2}$ B. N. Yeltsin Ural Federal University, 19 Mira St., Ekaterinburg, Russian Federation
}

a) (iD https://orcid.org/0000-0002-2083-5377, ه smirnov.sergy@bk.ru; ${ }^{\text {b) }}$ (D) https://orcid.org/0000-0003-0645-548X, 凶khotinov@yandex.ru; ${ }^{\mathrm{c})}$ (D) https://orcid.org/0000-0002-6508-6859, $\otimes$ mmm@imach.uran.ru;

d) $\otimes$ olga.polukhina @ chelpipe.ru; ${ }^{\mathrm{e})}$ 冈 farb.amo@r66.ru

*Corresponding author. E-mail: khotinov@ yandex.ru

Address for correspondence: Department of Heat Treatment and Physics of Metals, Yeltsin UrFU, 19, ul. Mira, Ekaterinburg, 620002, Russian Federation Tel.: +7-912-648-36-58

The plastic flow of the 08G2BM steel under tension is studied by the digital image correlation (DIC) method. The plastic strain field in a sample at different stages of the stress-elongation curve is shown. The strain tensor components $\varepsilon_{y y}$ along the load axis and the rate of its change $\dot{e}$ are evaluated quantitatively.

Keywords: stress-elongation curve, digital image correlation, inelastic strain field, plastic flow, strain rate.

\section{References}

1. Sutton M.A., Orteu J.-J., Schreier H.W. Image correlation for shape, motion and deformation measurements. Columbia, SC, USA, University of South Carolina, 2009, 364 p. DOI: $10.1007 / 978-0-387-78747-3$.

2. Vildeman V.E., Tretyakov M.P., Tretyakova T.V., Bulbovich R.V., Slovikov S.V., Babushkin A.V., Ilyinykh A.V., Lobanov D.S., Ipatova A.V. Eksperimentalnye issledovaniya svoistv materialov pri slozhnykh termomekhanicheskikh vozdeistviyakh [Experimental Research of Material Properties under Complex Thermomechanical Effects]. Moscow, Fizmatlit Publ., 2012, 204 p. (In Russian).

3. Renard K., Ryelandt S., Jacques P.J. Characterisation of the Portevin-Le Chatelier effect affecting an austenitic TWIP steel based on digital image correlation. Mat. Science and Eng. A, 2010, vol. 527, pp. 2969-2977. DOI: 10.1016/j.msea.2010.01.037.

4. Nagarajan S., Raghu N., Venkatraman B. Advanced imaging for early prediction and characterization of zone of Luders band nucleation associated with pre-yield microstrain. Mat. Science and Eng. A, 2013, vol. 561, pp. 203-211. DOI: 10.1016/j.msea.2012.10.082.

5. Ren Ch, Zhang X., Ji H., Zhan N., Qiao Zh. Effect of banded morphology and grain size on the tensile behavior of acicular ferrite in HSLA steel. Mat. Science and Eng. A, 2017, vol. 705, pp. 394-401. DOI: 10.1016/j.msea.2017.08.109.

6. Product-Manual for DaVis 8.1. Göttingen, Germany, La Vision GmbH, 2013, 338 p.

7. Makarov P.V. Mathematical theory of evolution of loaded solids and media. Phys. Mesomech., 2008, vol. 11, iss. 5-6, pp. 213-227. - DOI: 10.1016/j.physme.2008.11.002. 
Подана в журнал: 05.04.2018

УДК 620.172

DOI: $10.17804 / 2410-9908.2018 .3 .006-013$

\title{
ИССЛЕДОВАНИЕ МЕТОДОМ КОРРЕЛЯЦИИ ЦИФРОВЫХ ИЗОБРАЖЕНИЙ ПЛАСТИЧЕСКОГО ТЕЧЕНИЯ СТАЛИ ОВГ2БМ ПРИ РАСТЯЖЕНИИ
}

\author{
С. В. Смирнов ${ }^{1, \text { a) }}$, В. А. Хотинов $\left.{ }^{2,}\right)^{*}$, Д. И. Вичужанин ${ }^{1, \text { в) }}$ \\ О. Н. Полухина ${ }^{2, \text { г) }}$, В. М. Фарбер ${ }^{2, \text { य) }}$ \\ ${ }^{1}$ Институт машиноведения УрО РАН, ул. Комсомольская, 34, г. Екатеринбург, Российская Федераиия, \\ ${ }^{2}$ Уральский федеральный университет, ул. Мира, 19, г. Екатеринбург, Российская Федерация \\ a) iD https://orcid.org/0000-0002-2083-5377, $ه$ smirnov.sergy@bk.ru; ${ }^{\text {) }}$ (iD https://orcid.org/0000-0003-0645-548X, \\ هkhotinov@yandex.ru; ${ }^{\text {B) }}$ (iD https://orcid.org/0000-0002-6508-6859, $\otimes$ mmm@imach.uran.ru;

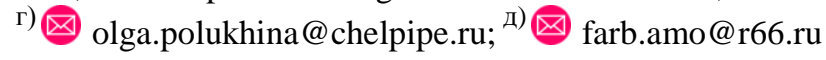 \\ *Ответственный автор. Электронная почта: khotinov@yandex.ru \\ Адрес для переписки: ул. Мира, 19, г. Екатеринбург, Российская Федерация \\ Тел.:+7-912-648-36-58
}

Методом корреляции цифровых изображений (КЦИ) изучено пластическое течение стали 08Г2БМ при растяжении. Показана картина распределения пластической деформации в образце на разных стадиях кривой растяжения. Проведена количественная оценка компо-

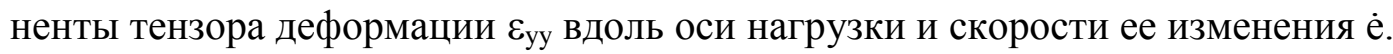

Ключевые слова: кривая растяжения, корреляция цифровых изображений, поле деформаций, пластическое течение, скорость деформации.

\section{1. Введение}

Магистральные трубопроводы эксплуатируются в достаточно жестких условиях, связанных со значительным перепадом температур в течение года, высокими внутренними давлениями и т.д. Поэтому для строительства магистральных трубопроводов используют высокопрочные стали классов прочности X70, X80, X90, X100. Такие стали имеют следующие характеристики: обладают высокой прочностью и пластичностью; высокой сопротивляемостью хрупкому разрушению при температурах до $-60{ }^{\circ} \mathrm{C}$; имеют высокую коррозионную стойкость. Очень важной является оценка механических свойств сталей для магистральных трубопроводов.

Современное испытательное оборудование для проведения механических испытаний позволяет автоматизировать процесс получения и обработки измеряемых данных, а также получать принципиально новую информацию о свойствах материалов. Это значительно расширяет возможности известных методик механических испытаний [1-2].

Так, в последнее время при испытаниях на растяжение активно используется метод корреляции цифровых изображений КЦИ (digital image correlation DIC), позволяющий на основе измерения полей перемещений материальных точек на поверхности образца проводить анализ поведения металла на разных стадиях деформации [3-5].

Цель настоящей работы - изучение особенностей пластического течения низкоуглеродистой микролегированной стали 08Г2БМ при растяжении с использованием метода корреляции цифровых изображений. 


\section{2. Материалы и методики исследования}

Материалом для исследования была выбрана сталь 08Г2БМ, используемая для сварных труб класса прочности Х80, следующего химического состава (мас. \%): 0,08 C; 1,85 Mn; 0,13 Mo; $\Sigma(\mathrm{Ti}-\mathrm{V}-\mathrm{Nb}) \approx 0,10 ; 0,17 \mathrm{Cu} ; 0,4 \mathrm{Si} ; 0,19 \mathrm{Cr} ; 0,22 \mathrm{Ni} ; 0,001 \mathrm{~S} ; 0,013 \mathrm{P}$. Стандартные плоские образцы для механических испытаний толщиной 3 мм, шириной 20 мм и длиной рабочей части 60 мм были вырезаны из стенки трубы диаметром 1420 мм и толщиной 27,7 мм в направлении, перпендикулярном оси трубы (направлению прокатки).

Растяжение с постоянной скоростью деформации е $=2,7 \cdot 10^{-4} \mathrm{c}^{-1}$ плоских образцов проводилось на универсальной испытательной машине Instron 8801, снабженной оптическим комплексом Strain Master для анализа полей перемещений и деформаций методом корреляции цифровых изображений (КЦИ). Технические характеристики комплекса Strain Master приведены в табл. 1. Для получения изображения картины деформации на поверхность образца при помощи спрейера наносили капельки краски (серый фон с дисперсными черными вкраплениями), что позволило при калибровке образца разбить ее на множество ячеек для анализа методом кросс-корреляции (оценка смещения точки поверхности в пределах каждой элементарной ячейки в двух последовательных кадрах) [6].

Таблица 1 - Технические характеристики системы StrainMaster

\begin{tabular}{|l|l|}
\hline Разрешение CCD видеокамеры & $1600 \times 1200$ пикселей \\
\hline Динамический диапазон ССD видеокамеры & 14 бит \\
\hline Скорость затвора СCD видеокамеры & 1 мкс \\
\hline Частота кадров & 30 кадров/с \\
\hline Минимальный межкадровый интервал & 110 нс \\
\hline Размер пикселя & 7,4 мкм \\
\hline Точность оценки изображения & до 0,01 пикселя \\
\hline Объективы & $24-85 \mathrm{Mм} \mathrm{F/2.8} \mathrm{-4}$ \\
\hline
\end{tabular}

Полученные в процессе нагружения смещения точек на поверхности образца программно обрабатывались с целью получения векторных полей перемещений и компонент тензора деформации на поверхности испытываемого образца [6]:

$$
\varepsilon_{\mathrm{ij}}=\frac{1}{2}\left(\frac{\partial \mathrm{V}_{\mathrm{i}}}{\partial \mathrm{r}_{\mathrm{j}}}+\frac{\partial \mathrm{V}_{\mathrm{j}}}{\partial \mathrm{r}_{\mathrm{i}}}\right)
$$

где $\mathrm{V}_{\mathrm{i}}$ - векторная компонента смещения точки вдоль направления $\mathrm{i}(\mathrm{i}=\mathrm{x}, \mathrm{y}, \mathrm{z}) ; \mathrm{r}_{\mathrm{j}}-$ пространственная ось по направлению $\mathrm{j}(\mathrm{j}=\mathrm{x}, \mathrm{y}, \mathrm{z})$.

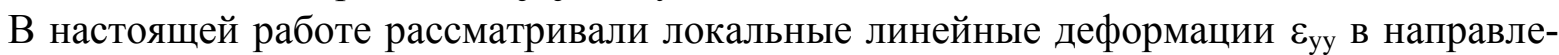
нии оси растяжения (по длине образца).

\section{3. Результаты эксперимента и их обсуждение}

Диаграмма растяжения образца имеет вид гладкой параболической кривой $\left(\sigma_{0,2}=610 \mathrm{MПа,}\right.$ $\sigma_{\mathrm{B}}=670 \mathrm{MПа,} \delta=12,5 \%$ ) (рис. 1 a). При этом с помощью метода КЦИ можно проследить пластическое течение металла на всех стадиях кривой растяжения (рис. 1 б). Так, при переходе от упругой к пластической деформации в точке $1(\delta=0,2 \%)$ смещения на поверхности металла крайне малы $\varepsilon_{\text {уу }}=0,4 \ldots 0,6$ \% и наблюдаются по всей длине образца. На поле деформаций видна легкая полосчатость, связанная, по-видимому, с локальной микропластической деформацией в благоприятно ориентированных к оси растяжения For citation: The digital image correlation method applied to studying the plastic flow of the 08G2BM steel under tension / S. V. Smirnov, V. A. Khotinov, D. I. Vichuzhanin, O. N. Polukhina, V. M. Farber // Diagnostics, Resource and Mechanics of materials and structures. - 2018. - Iss. 3. - P. 6-13. - DOI: 10.17804/2410-9908.2018.3.006-013. 
участках. Наблюдаемые полосы шириной 3-5 мм расположены к оси нагружения под углом $30-45^{\circ}$, а степень деформации в них выше на $\sim 0,2 \%$ по сравнению с другими участками.

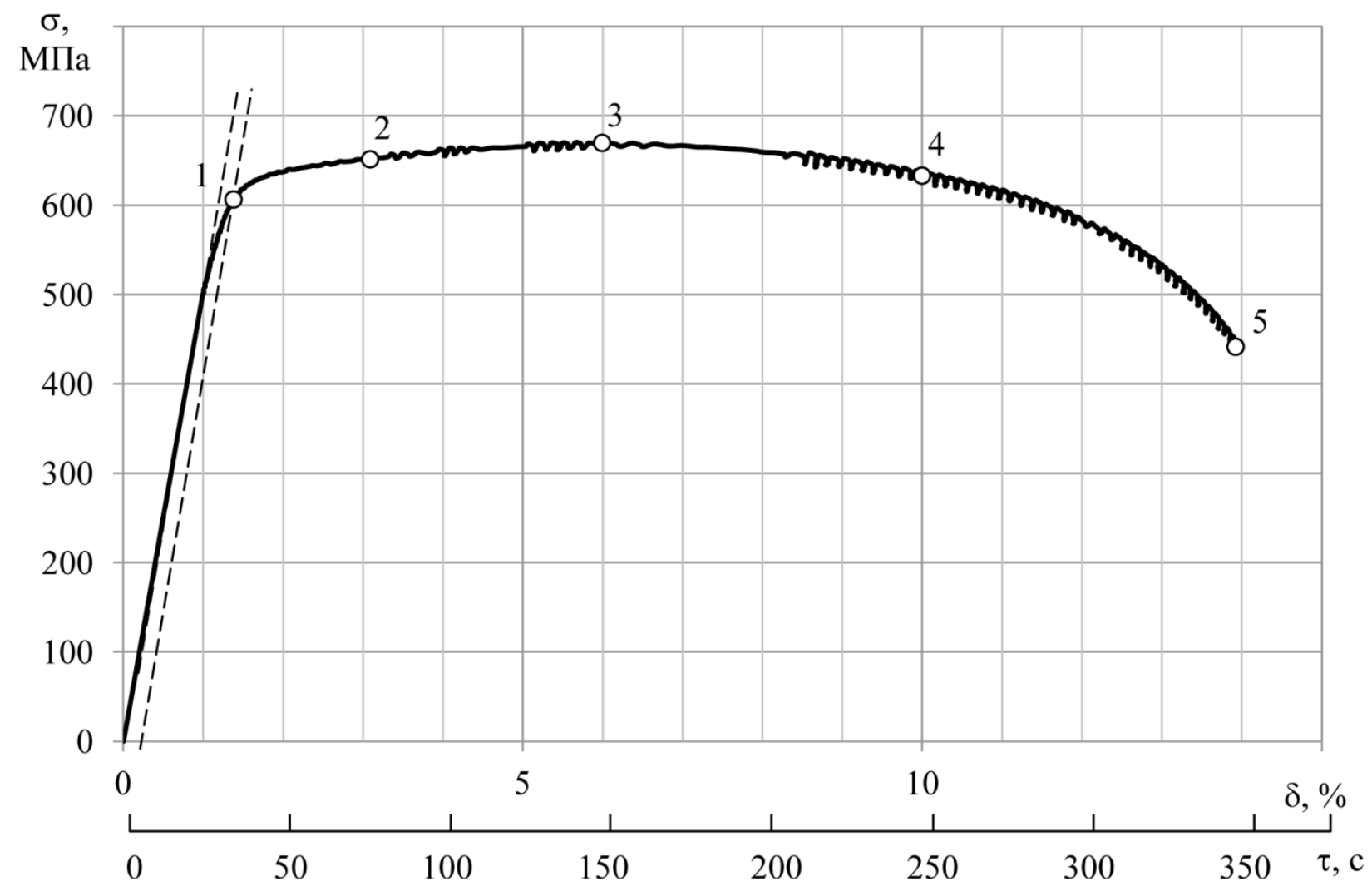

$a$

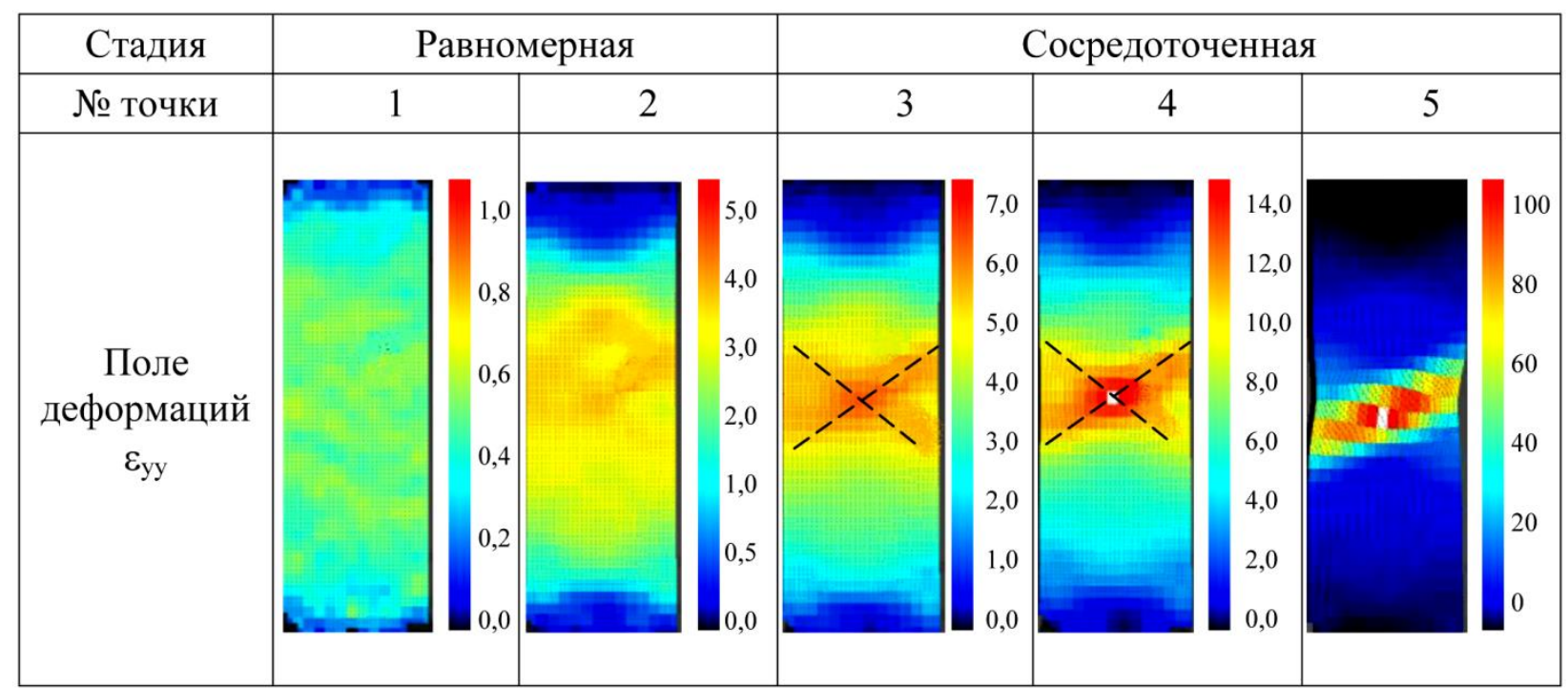

$\sigma$

Рис. 1. Диаграмма растяжения стали 08Г2БМ (a) и поля деформаций компоненты $\varepsilon_{y у}$ на различных стадиях пластической деформации (б)

C ростом деформации образца (точка 2 соответствует равномерной стадии пластического течения) область пластического течения начинает сужаться (до 40 мм) при

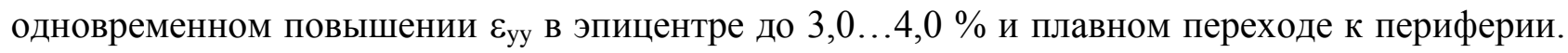

For citation: The digital image correlation method applied to studying the plastic flow of the 08G2BM steel under tension / S. V. Smirnov, V. A. Khotinov, D. I. Vichuzhanin, O. N. Polukhina, V. M. Farber // Diagnostics, Resource and Mechanics of materials and structures. - 2018. - Iss. 3. - P. 6-13. - DOI: 10.17804/2410-9908.2018.3.006-013. 
Такое поведение характерно для исследуемого образца на всем протяжении равномерной стадии деформации вплоть до момента, пока в центре образца не станет заметно формирование концентратора напряжений (точка 3 на рис. 1). При этом видно, что пластическое течение металла около концентратора осуществляется наиболее интенсивно $\left(\varepsilon_{\mathrm{yy}}=5 \ldots 6 \%\right)$ в виде двух взаимно перпендикулярных полос красного цвета, расположенных под углом $\sim 45^{\circ}$ к оси растяжения (выделены пунктиром).

Дальнейшая деформация образца приводит к ее локализации и образованию шейки, смещения объемов металла ( $\left.\varepsilon_{\text {yу }}=13 \ldots 15 \%\right)$ происходят только в областях, прилегающих к ней (точка 4 на рис. 1). Затем интенсивное пластическое течение локализуется в шейке в виде узкого максимума $\left(\varepsilon_{\mathrm{yy}}=80 \ldots 100 \%\right)$ шириной $\sim 5$ мм (точка 5 на рис. 1$)$,

Проследить продольную деформацию в растягиваемом образце позволяет и анализ

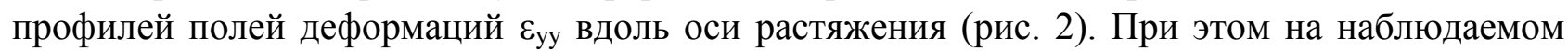
поле можно выделить разные по интенсивности области пластического течения - активную зону (АЗ), в области которой идет интенсивное пластическое течение, и окружающие ее релаксационные зоны (Р3), в которых вследствие инструментальных факторов (близость головок образца, его соосность и др.) и физических факторов деформация металла значительно меньше.

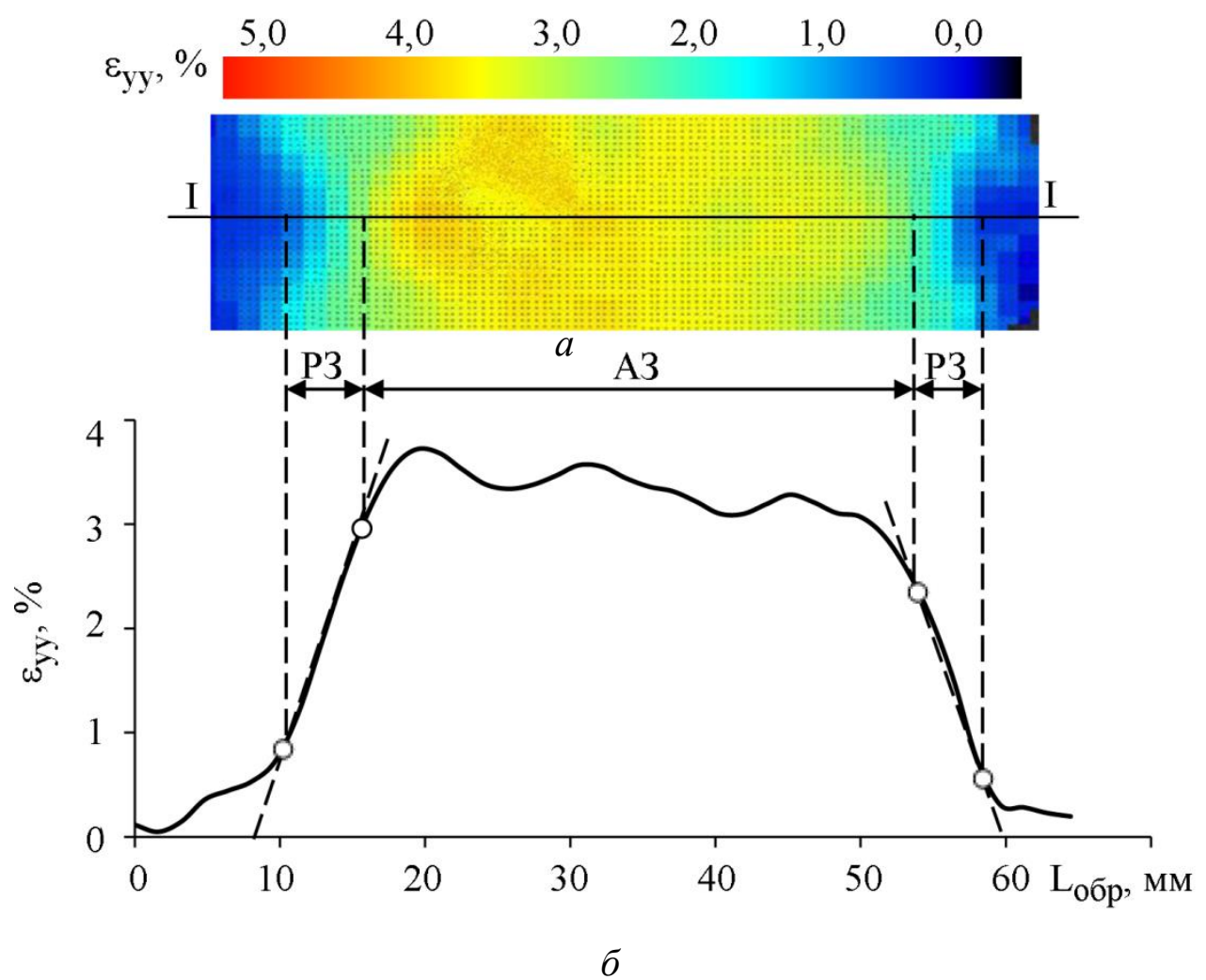

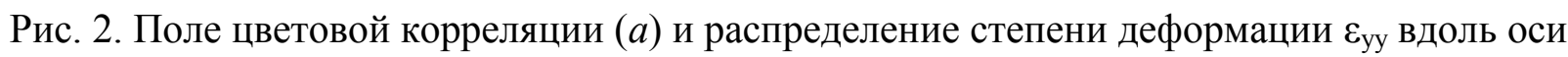
образца по трассе I-I (б) на равномерной стадии кривой растяжения:

АЗ - активная зона; Р3 - релаксационная зона

Релаксационная зона на профиле поля деформаций представлена в виде двух ниспадающих линейных участков по обе стороны от пика, активную зону можно определить как область между точками отклонения кривой профиля от выделенных линейных участков (рис. 2). На упругой стадии кривой растяжения обратимые смещения металла малы $\left(\varepsilon_{\mathrm{yy}} \leq 0,5 \%\right)$ и равномерно распределены по всей длине образца. 


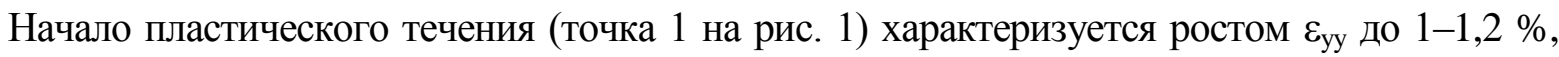
а также разделением области пластического течения на активную и релаксационные зоны. При этом в активной зоне начинают все более четко формироваться три максимума, расположенных на одинаковом расстоянии между собой $\left(\mathrm{M}_{1}, \mathrm{M}_{2}, \mathrm{M}_{3}\right.$ на рис. $\left.3 a\right)$. Дальнейшее равномерное пластическое течение приводит к возрастанию деформации во всей активной зоне и в отдельных максимумах.
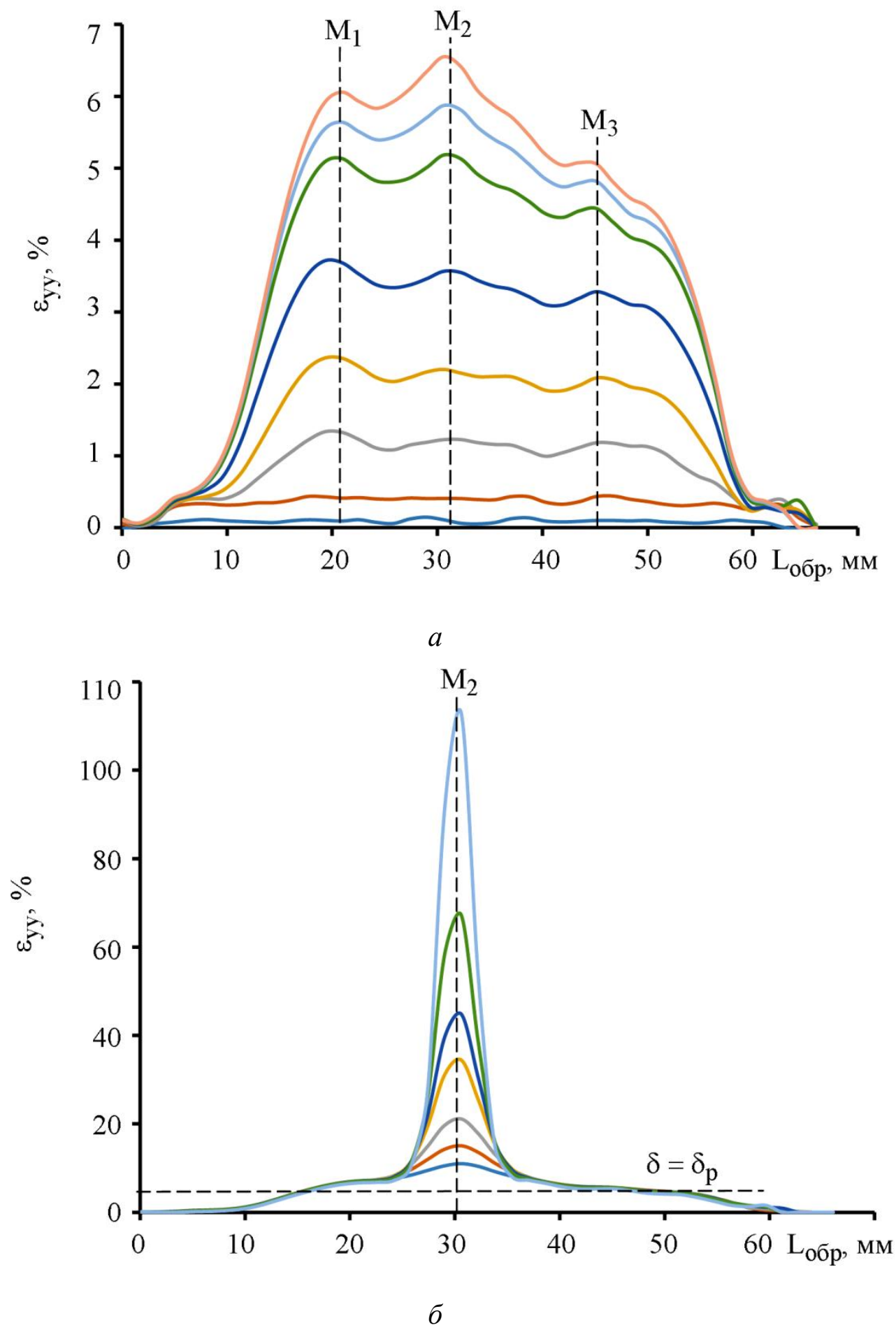

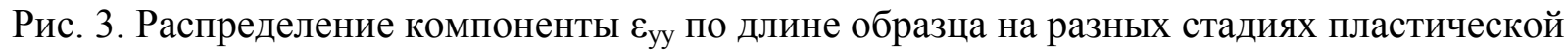
деформации: $a$ - равномерная; $\sigma$ - сосредоточенная 
При локализации пластического течения в шейке средний максимум $\mathrm{M}_{2}$, лежащий на середине длины образца, как бы поглощает соседние максимумы и пластическая деформация протекает исключительно в нем (рис. 3 б).

Представляет интерес оценить изменение скорости пластического течения (скорость деформации ё) в процессе растяжения образца. Для расчета еं выделяли максимум компоненты смещений $\varepsilon_{\text {уу }}{ }^{\max }$ на профиле в любой момент времени, а затем определяли ее величину как

$$
\dot{e}=\frac{\Delta \varepsilon_{y y}^{\max }}{\Delta \tau},
$$

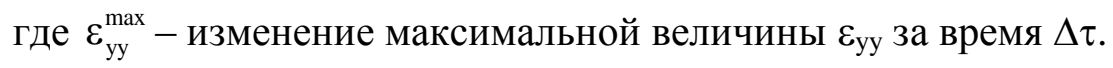

На зависимости $\dot{\mathrm{e}}=f(\tau)$ наблюдается два линейных участка, соответствующих равномерной и сосредоточенной стадиям деформации (рис. 4).

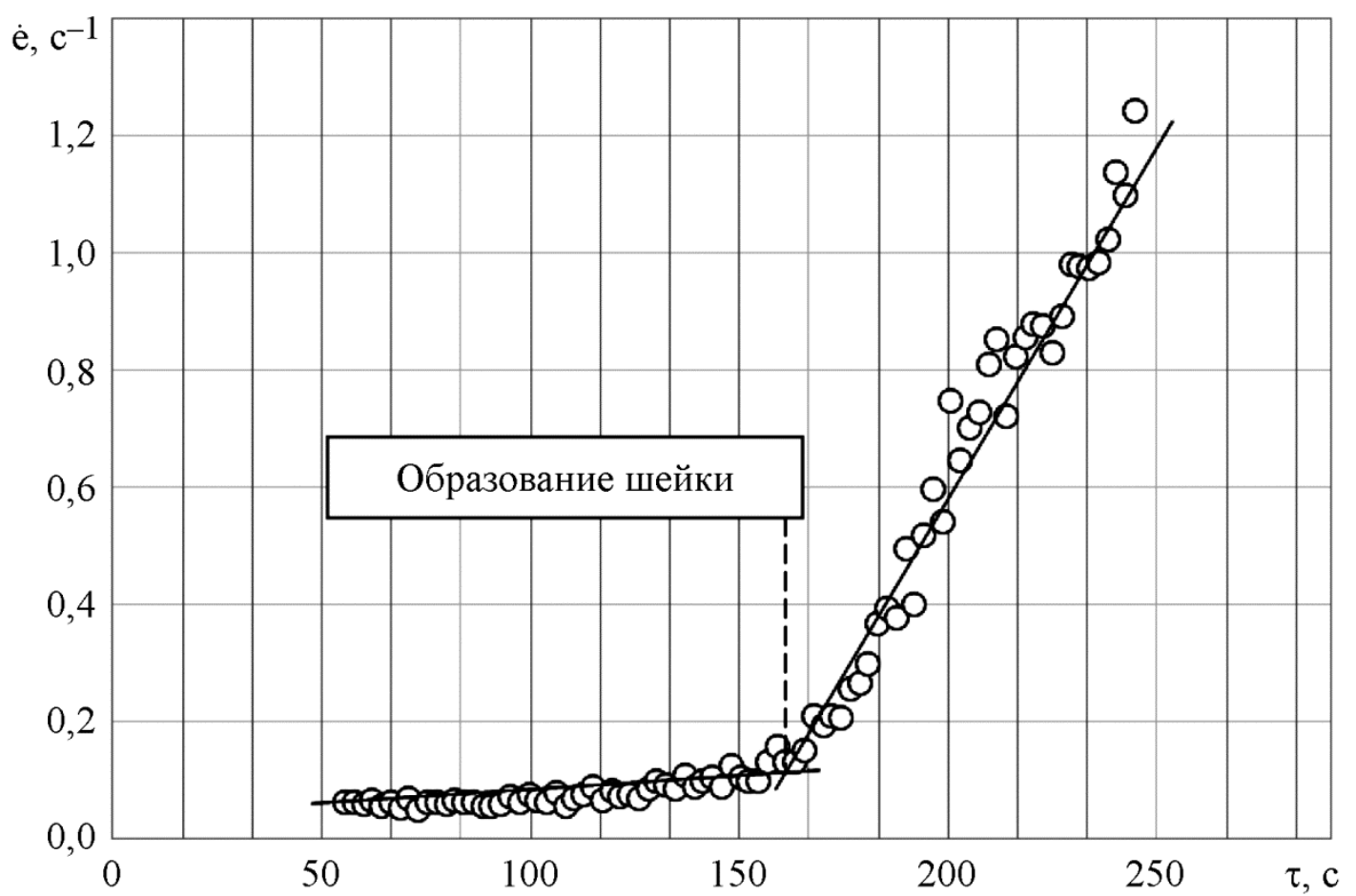

Рис. 4. Интенсивность нарастания $\varepsilon_{\text {уу }}$ (скорость деформации ё) вдоль центра образца

Видно, что на стадии равномерной деформации (до $\tau \approx 150 \mathrm{c}$ ) ее скорость незначительна и не превышает $0,1 \mathrm{c}^{-1}$, однако после локализации деформации в шейке е резко возрастает по линейному закону. Медленный квазистатический режим пластического течения сменяется катастрофическим. Это является общим свойством самоорганизации деформационных процессов в нагружаемых твердых телах [7].

\section{4. Заключение}

Применение метода корреляции цифровых изображений позволяет детально описать пластическое течение металла на разных стадиях кривой растяжения. Так, на примере стали 08Г2БМ обнаружено, что при одноосном растяжении неоднородность распределения деформации по длине образца возникает уже в начале пластического течения вблизи $\sigma_{0,2}\left(\varepsilon_{\mathrm{yy}}=0,2-0,6 \%\right)$ в виде полос под углом $\sim 30-45^{\circ}$ к оси нагружения.

For citation: The digital image correlation method applied to studying the plastic flow of the 08G2BM steel under tension / S. V. Smirnov, V. A. Khotinov, D. I. Vichuzhanin, O. N. Polukhina, V. M. Farber // Diagnostics, Resource and Mechanics of materials and structures. - 2018. - Iss. 3. - P. 6-13. - DOI: 10.17804/2410-9908.2018.3.006-013. 
На равномерной стадии пластического течения эта неоднородность усиливается - на

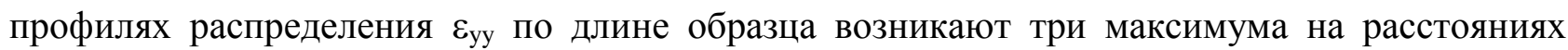
$\Delta \mathrm{L}_{\text {обр }}=10-15$ мм, развитие которых продолжается вплоть до образования шейки. На сосредоточенной стадии деформации центральный максимум как бы поглощает соседние, пластическое течение локализуется в нем, а скорость деформации по сравнению с равномерной стадией повышается на порядок ( $\mathrm{c} \dot{\mathrm{e}}=0,1$ до $\left.1,2 \mathrm{c}^{-1}\right)$.

Работа выполнена в УрФУ при финансовой поддержке постановления № 211 Правительства Российской Федераџии, контракт № 02.A03.21.0006, а также в ИМАШ УрО РАН в рамках темь государственного задания № 0391-2016-0004. Испьттания проведены с использованием оборудования ЦКП УрФУ и ЦКП «Пластометрия» ИМАШ УрО РАН.

\section{Литература}

1. Sutton M.A., Orteu J.-J., Schreier H.W. Image correlation for shape, motion and deformation measurements. - Columbia, SC, USA: University of South Carolina, 2009. - 364 p. DOI: $10.1007 / 978-0-387-78747-3$.

2. Экспериментальные исследования свойств материалов при сложных термомеханических воздействиях / В. Э. Вильдеман, М.П. Третьяков, Т.В. Третьякова, Р.В. Бульбович, С.В. Словиков, А.В. Бабушкин, А.В. Ильиных, Д.С. Лобанов, А.В. Ипатова. -М. : Физматлит, 2012. - $204 \mathrm{c}$.

3. Renard K., Ryelandt S., Jacques P.J. Characterisation of the Portevin-Le Chatelier effect affecting an austenitic TWIP steel based on digital image correlation // Mat. Science and Eng. A. 2010. - Vol. 527. - P. 2969-2977. - DOI: 10.1016/j.msea.2010.01.037.

4. Nagarajan S., Raghu N., Venkatraman B. Advanced imaging for early prediction and characterization of zone of Luders band nucleation associated with pre-yield microstrain // Mat. Science and Eng. A. - 2013. - Vol. 561. - P. 203-211. - DOI: 10.1016/j.msea.2012.10.082.

5. Effect of banded morphology and grain size on the tensile behavior of acicular ferrite in HSLA steel / Ch. Ren, X. Zhang, H. Ji, N. Zhan, Zh. Qiao // Mat. Science and Eng. A. - 2017. Vol. 705. - P. 394-401. - DOI: 10.1016/j.msea.2017.08.109.

6. Product-Manual for DaVis 8.1. - Göttingen, Germany:La Vision GmbH. - 2013. - 338 p.

7. Makarov P.V. Mathematical theory of evolution of loaded solids and media // Phys. Mesomech. - 2008. - Vol. 11, iss, 5-6. - P. 213-227. - DOI: 10.1016/j.physme.2008.11.002. 